\title{
Characteristics of Refractory Gastroesophageal Reflux Disease (GERD) Symptoms - Is Switching Proton Pump Inhibitors Based on the Patient's CYP2C19 Genotype an Effective Management Strategy?
}

\author{
Toshihisa Takeuchi ${ }^{1}$, Kazuhiro Oota ${ }^{1}$, Satoshi Harada ${ }^{1}$, Shoko Edogawa ${ }^{1}$, Yuichi Kojima ${ }^{1}$, \\ Makoto Sanomura ${ }^{2}$, Masahiro Sakaguchi ${ }^{3}$, Katsuyoshi Hayashi ${ }^{4}$, Yasushi Hongoh ${ }^{5}$, \\ Tsukasa Itabashi ${ }^{6}$, Hidehiro Kitae ${ }^{7}$, Masahiro Hoshimoto ${ }^{8}$, \\ Nozomi Takeuchi ${ }^{9}$ and Kazuhide Higuchi ${ }^{1}$
}

\begin{abstract}
Objective We investigated factors related to proton pump inhibitor (PPI) -refractory gastroesophageal reflux disease (GERD) symptoms, particularly with respect to acid, the CYP2C19 genotype and psychological aspects.

Methods Patients with an Frequency Scale for the Symptoms of GERD (FSSG) score of $\geq 8$ after the initial treatment were switched to therapy with rabeprazole at a dose of $20 \mathrm{mg}$ once daily for eight weeks. We investigated the rate of improvement in PPI-refractory GERD symptoms, background factors, the Hospital Anxiety and Depression Scale (HADS) score and the CYP2C19 genotype.

Patients Sixty patients endoscopically diagnosed with reflux esophagitis within the past six months who had received omeprazole at a dose of $20 \mathrm{mg}$ once daily for eight weeks or longer were enrolled.

Results In $71.6 \%$ of the patients, the FSSG score decreased to $<8$ after treatment with omeprazole at a dose of $20 \mathrm{mg}$ once daily for $\geq 8$ weeks, resulting in improvements in their GERD symptoms. Significant factors related to omeprazole-refractory GERD symptoms included a longer disease duration $(\mathrm{p}=0.0004)$ and higher HADS score $(\mathrm{p}=0.01)$. Among the omeprazole-refractory cases, only $23.5 \%$ of the patients showed symptom improvement after switching to rabeprazole. There were no significant differences in the average scores for FSSG ( $\mathrm{p}=0.089)$ or HADS $(\mathrm{p}=0.182)$, before or after the drug change. A total of $92 \%$ of the rabeprazole poor responders were homo/hetero extensive metabolizers for the CYP2C19 genotype.

Conclusion Our findings suggest that switching the PPI from omeprazole (20 $\mathrm{mg}$ once daily) to rabeprazole (20 mg once daily) is not a significant effective therapeutic strategy for improving PPI-refractory GERD symptoms, taking into consideration possible psychometric factors and patients who require stronger acid suppression than that achieved with a double dose of PPIs for PPI-refractory GERD symptoms.
\end{abstract}

Key words: omeprazole, rabeprazole, CYP2C19 genotype, gastroesophageal reflux disease, PPI-refractory GERD symptoms

(Intern Med 54: 97-105, 2015)

(DOI: 10.2169/internalmedicine.54.3412)

\footnotetext{
${ }^{1}$ The Second Department of Internal Medicine, Osaka Medical College, Japan, ${ }^{2}$ Hokusetsu General Hospital, Japan, ${ }^{3}$ Moriguchi Keijinkai Hospital, Japan, ${ }^{4}$ Ishikiri Seiki Hospital, Japan, ${ }^{5}$ Hirakata City Hospital, Japan, ${ }^{6}$ Hanwasumiyoshi General Hospital, Japan, ${ }^{7}$ Aoyama Hospital, Japan, ${ }^{8}$ Seikeikai Hospital, Japan and ${ }^{9}$ Settsu Clinic, Japan

Received for publication June 5, 2014; Accepted for publication July 13, 2014

Correspondence to Dr. Toshihisa Takeuchi, in2097@ poh.osaka-med.ac.jp
} 


\section{Introduction}

Gastroesophageal reflux disease (GERD) is a condition that damages the esophageal mucosa via the regurgitation of stomach contents, primarily gastric acid, into the esophagus. Global attention has been focused on GERD as a disease since the 1980's, and the Montreal Definition (1) was released in 2006 as an international consensus on this disorder. Subsequently, the Asian-Pacific Consensus (2) in the Asia-Pacific region, and ACG Guidelines (3) of the American College of Gastroenterology (ACG) in the USA were formulated as international treatment guidelines for GERD.

The incidence of reflux esophagitis (RE) is increasing (4) in Japan in association with aging of the population, Westernization of the diet, a decrease in the rate of Helicobacter pylori (H. pylori) infection, etc. As in the overseas guidelines, the GERD treatment guidelines (5) compiled by the Japanese Society of Gastroenterology recommend the use of proton pump inhibitors (PPIs) as the first choice drug both for RE and non-erosive reflux disease (NERD).

The standard dose of PPIs is administered in most patients with GERD; however, GERD symptoms do not exhibit remission in some patients, and several treatment methods may be applied in cases in which the therapeutic effect of standard-dose PPI therapy is insufficient. Concerning drug therapy, several investigations have been published regarding the efficacy of changing the dose or dividing the administration of PPIs (6-8), as well as the concomitant use of other drugs, including histamine $\mathrm{H}_{2}$-receptor antagonists $\left(\mathrm{H}_{2} \mathrm{RAs}\right)(9)$ and prokinetic agents (10). In addition, switching between PPI medications is another treatment choice.

Currently, four PPIs are clinically available in Japan, including omeprazole, lansoprazole, rabeprazole and esomeprazole. However, the findings of reports of differences in clinical effect between PPIs vary, and the choice of drug and dose depend on the clinical experience of the physician. As a result, there is currently no established evidence supporting a standard treatment regimen.

In this study, we investigated the background factors of PPI-refractory patients with GERD symptoms who did not respond sufficiently to treatment with omeprazole at a dose of $20 \mathrm{mg}$ once daily, the standard drug and dose of PPI therapy in Japan, and evaluated the therapeutic effect of switching to rabeprazole at a dose of $20 \mathrm{mg}$ once daily, double the recommended dose in Japan. In addition, psychometric factors were considered as a cause of a treatment refractory status, as an association between GERD symptoms and anxiety or depression has been reported in some studies $(11,12)$.

\section{Materials and Methods}

\section{Patients}

Of the patients who visited institutions from August 2010 to August 2012, those 20 years of age or older who were endoscopically diagnosed with RE associated with erosion of grade A to D according to the LA classification within the past six months and had received omeprazole at a dose of $20 \mathrm{mg}$ once daily for eight weeks or longer were enrolled consecutively at each site.

Patients were excluded based on the following criteria: (i) a history or complications of Zollinger-Ellison syndrome, inflammatory bowel disease or irritable bowel syndrome, (ii) a history of gastroenterectomy or vagotomy, (iii) complications of peptic ulcers, (iv) serious hepatic, renal or cardiac disease, (v) a history of $H$. pylori eradication therapy within two months prior to the start of the study, (vi) treatment with the continuous administration of any drugs known to interact with the study drugs.

The study protocol was conducted according to the Helsinki Declaration, with written informed consent obtained from the patients after sufficiently explaining the purpose, methods, merits and possible risks of the study. Approval of the Institutional Review Board of each hospital was obtained prior to initiation of the study.

\section{Study design and method}

This study was designed as a multicenter, single-arm, open label study.

Fig. 1 shows the study procedure. Patients who satisfied the inclusion criteria completed the following questionnaires: the Frequency Scale for the Symptoms of GERD (FSSG) (13) and Hospital Anxiety and Depression Scale (HADS) (14). The HADS has been previously validated and consists of seven questions for anxiety and seven questions for depression. Each item is answered on a 4-point (0-3) scale. The score for each subcategory (anxiety and depression) ranges from $0-21$, as follows: without anxiety/depression (0-7), suspected anxiety/depression (8-10), with anxiety/depression $(11<)$.

Excluding PPIs, the concomitant use of drugs for the digestive system was allowed only in cases in which the treatment had been initiated prior to the switch to rabeprazole; no changes in the dose or agent were allowed during the study period.

\section{Endpoints}

The primary endpoint was the identification of background factors related to the therapeutic effect of the oral administration of omeprazole at a dose of $20 \mathrm{mg}$ once daily for $\geq 8$ weeks. The rate of symptom improvement was defined as an FSSG score of $<8$ points. The secondary endpoint was the identification of factors of a poor response to rabeprazole treatment based on the same definition for the FSSG score. Furthermore, the CYP2C19 genotype and changes in the FSSG and HADS scores from before to after the oral administration of rabeprazole were investigated.

\section{Statistical methods}

The rate of symptom improvement was presented descrip- 


\section{Patients:}

RE patients taking omeprazole $20 \mathrm{mg}$

once daily for $\geq \mathbf{8}$ weeks

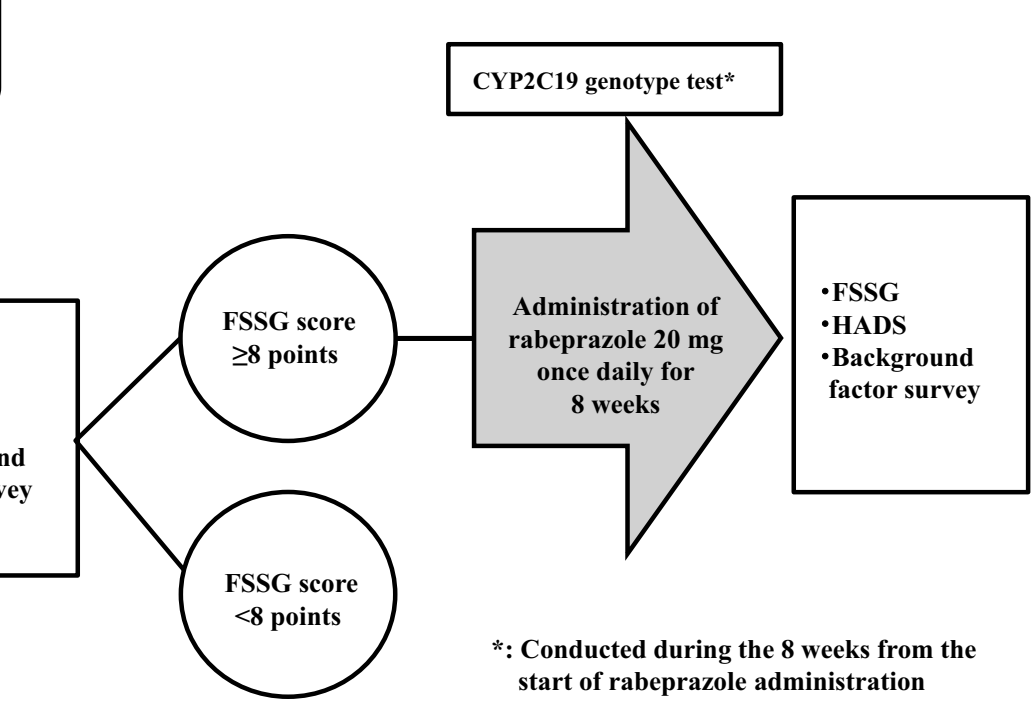

Figure 1. Study design. Factors related to the therapeutic response to therapy with orally administered omeprazole ( $20 \mathrm{mg}$ once daily) were investigated in the RE patients receiving treatment with omeprazole at a dose of $20 \mathrm{mg}$ once daily for $\geq 8$ weeks and who satisfied the enrollment criteria. In addition, changes in the FSSG and HADS scores were investigated. Patients with an FSSG score of $\geq 8$ points were managed as being omeprazole-refractory, and the treatment regimen was changed to the oral administration of rabeprazole at a dose of $20 \mathrm{mg}$ once daily for eight additional weeks. CYP2C19 gene tests were conducted during the rabeprazole treatment period, and the same factors as those investigated during the treatment period with omeprazole ( $20 \mathrm{mg}$ once daily) were again investigated, as well as changes in the FSSG and HADS scores after the completion of rabeprazole therapy.

tively. Background factors related to an unsuccessful response to omeprazole and/or rabeprazole were analyzed according to the $\chi^{2}$ test, with a significance level of $5 \%$.

Changes in the mean FSSG and HADS scores were analyzed according to the two-sided paired $t$-test, with a significance level of $5 \%$.

\section{Sample size}

A sample size of approximately 100 patients was determined to be required for the investigation of background factors (approximately 10 factors) with respect to the primary endpoint (the treatment response to omeprazole at a dose of $20 \mathrm{mg}$ once daily for $\geq 8$ weeks) (15). Regarding the required number of patients for the secondary endpoint (the assessment of the rate of improvement in the FSSG scores from before to after treatment with rabeprazole at a dose of $20 \mathrm{mg}$ once daily), the proportion of subjects demonstrating a treatment response to therapy with omeprazole at a dose of $20 \mathrm{mg}$ once daily for $\geq 8$ weeks (proportion of those with a comprehensive FSSG score of $<8$ points) was assumed to be $60 \%$. Meanwhile, a total of 93 patients was required for the estimation of the confidence interval within a range of \pm $10 \%$. Accordingly, the target number of patients was set at 100.

Results

\section{Patient background factors and flowchart}

A total of 60 patients, including 32 men and 28 women, were enrolled in this study. The mean patient age was $61.9 \pm$ 11.2 years. A total of 17 patients smoked and 19 drank alcohol. Thirty patients had a disease duration between $\geq 2$ and $<12$ months.

Fifty patients were negative for $H$. pylori infection. With respect to RE, 29 patients had Grade A disease, 25 patients had Grade B disease, five patients had Grade C disease and one patient had Grade D disease according to the LA classification.

Fig. 2 shows the patient flowchart. A total of 17 patients were switched from omeprazole (20 mg once daily) to rabeprazole (20 mg once daily) due to a lack of therapeutic effect.

\section{Primary endpoint}

Table 1 shows the therapeutic effect of treatment with omeprazole at a dose of $20 \mathrm{mg}$ once daily for $\geq 8$ weeks according to the patients' background factors. The longer the duration of disease ( $p=0.0004, \chi^{2}$ test), the larger the number of patients found to be refractory to omeprazole therapy 


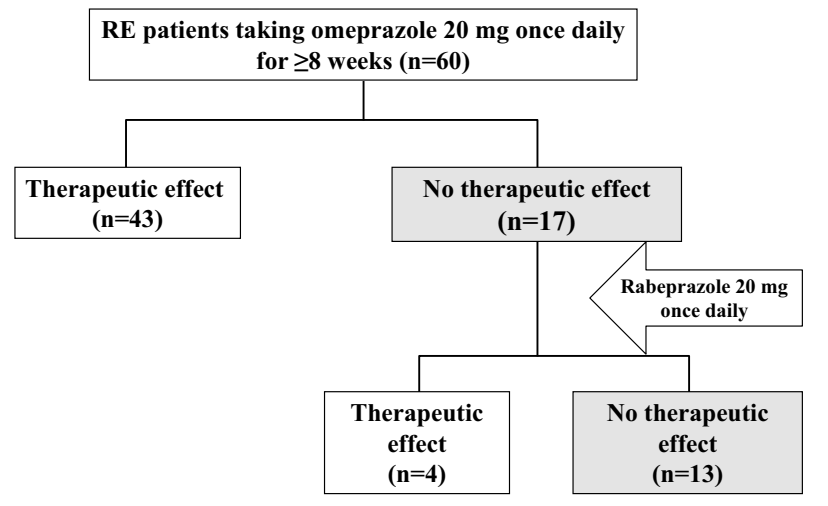

Figure 2. Patient flowchart. The therapeutic effect was investigated based on an FSSG score of $<8$ and $\geq 8$ points following the administration of oral omeprazole at a dose of $20 \mathrm{mg}$ once daily for $\geq 8$ weeks as being present (treatment response cases) or absent (treatment refractory cases), respectively, with a symptom improvement rate of $71.6 \%$. The oral administration of rabeprazole at a dose of $20 \mathrm{mg}$ once daily for eight weeks in 17 patients whose treatment with omeprazole $(20 \mathrm{mg}$ once daily) was judged to be ineffective (poor responders) did not show any improvements in symptoms.

at a dose of $20 \mathrm{mg}$ once daily. The subjects who tested negative for $H$. pylori infection (NS, $\mathrm{p}=0.159, \chi^{2}$ test) and did not have any complications $\left(N S, \mathrm{p}=0.076, \chi^{2}\right.$ test) tended to be more refractory to treatment; however, no other factors exhibited a significant difference.

Based on the results for the HADS score, the more severe the anxiety and/or depression, the greater the likelihood of being refractory to treatment with omeprazole at a dose of $20 \mathrm{mg}$ once daily ( $\mathrm{p}=0.01, \chi^{2}$ test) (Fig. 3 ). In addition, a significant positive correlation was observed between the FSSG and HADS score $(\mathrm{p}=0.00002, \mathrm{R}=0.562$, Spearman's rank correlation coefficient) (Fig. 4).

Among the patients with an FSSG score of $<8$ points, defined as achieving remission of GERD symptoms, the rate of symptom improvement associated with treatment with omeprazole at a dose of $20 \mathrm{mg}$ once daily was $71.6 \%$ (43/ 60 patients), while that associated with treatment with rabeprazole at a dose of $20 \mathrm{mg}$ once daily for eight weeks was $23.5 \%$ (4/17 patients).

\section{Secondary endpoint}

Treatment with rabeprazole at a dose of $20 \mathrm{mg}$ once daily did not correlate with any significant differences in the therapeutic effect when assessed according to the severity of anxiety and/or depression. In this regard, 92\% (12/13) of the rabeprazole treatment poor responders were homo/hetero extensive metabolizers (EM) of the CYP2C19 genotype (Table 2). Furthermore, no significant differences were observed in the FSSG or HADS score before or after the administration of rabeprazole ( $20 \mathrm{mg}$ once daily) $(\mathrm{p}=0.089, \mathrm{p}=$ 0.182 , respectively, paired $t$-test) (Fig. 5).

\section{Discussion}

In this study, the percentage of patients with an FSSG score of $\geq 8$ and omeprazole-refractory symptoms was $28.3 \%$ (17/60 patients), and, even after switching to the double dose of rabeprazole of $20 \mathrm{mg}$ once daily, no improvements in symptoms were observed in more than $75 \%$ of the patients.

Some reports have demonstrated that $10 \%$ to $20 \%$ of RE patients display resistance to PPI treatment (16-18); however, this finding is based on the rate of endoscopic healing, and it has also been reported that the number of patients with persistent symptoms is greater than recognized (19).

In a systematic review of seven trials comparing PPIs with a placebo, the rate of GERD symptom improvement after four weeks of treatment with a PPI was found to be $55.5 \%$ in patients with $\mathrm{RE}$ and $36.7 \%$ in patients with NERD, with a significant difference $(\mathrm{p}<0.0001)(20)$. Therefore, RE patients, who experience a longer duration of exposure to esophageal acid, obtain a higher symptom improvement rate with PPI treatment than NERD patients and that a higher percentage of NERD patients are refractory to PPI therapy.

The present study included patients with RE, which develops due to acid regurgitation, and therefore differs from studies of NERD patients in terms of background factors. Few reports have evaluated the degree of symptom improvement achieved by switching PPIs in patients whose symptoms persist after PPI treatment for $\geq 8$ weeks, as in this study. In order to fully evaluate the treatment effects, we switched to rabeprazole at a dose of $20 \mathrm{mg}$ once daily (double dose), which has a stronger acid-suppressing effect.

Comparing rabeprazole with omeprazole and lansoprazole, there were fewer differences in metabolic ability between the poor metabolizer (PM) and EM groups, and CYP2C19 contributed less to metabolic clearance. Therefore, rabeprazole is considered to be affected by CYP2C19 to a lower degree. In one study that evaluated the rate of recurrence of GERD symptoms in RE patients receiving maintenance therapy with omeprazole (20 mg once daily), lansoprazole (15 $\mathrm{mg}$ once daily) or rabeprazole (10 $\mathrm{mg}$ once daily) for six months, the recurrence rate was $25 \%$ in the omeprazole group, $30.8 \%$ in the lansoprazole group and $4.4 \%$ in the rabeprazole group; the recurrence rate in the rabeprazole group was significantly lower than that observed in the other two groups (21). In addition, the rate of recurrence according to the CYP2C19 genotype was $10.9 \%$ in the heterozygous EM group and $5.6 \%$ in the PM group, compared to $38.5 \%$ in the homozygous EM group. The authors pointed out that the presence of homozygous EM patients in the Japanese population reduces the clinical effects of PPIs.

In the present study, an improvement in symptoms was observed after switching to the double dose of rabeprazole (20 mg once daily) in $23.5 \%$, less than one-quarter, of the patients. Two possibilities may be considered based on this 
Table 1. Therapeutic Effect by Background Factor after Treatment with Omeprazole at a Dose of 20 mg Once Daily for $\geq 8$ Weeks

\begin{tabular}{|c|c|c|c|c|c|}
\hline Backgrou & d factors & $\begin{array}{l}\text { Omeprazole } \\
\text { responders }\end{array}$ & $\begin{array}{c}\text { Omeprazole } \\
\text { refractory cases }\end{array}$ & Total & p value (unknown \\
\hline \multirow[t]{2}{*}{ Gender } & Male & 23 & 9 & 32 & NS \\
\hline & Female & 20 & 8 & 28 & \\
\hline \multirow[t]{7}{*}{$\overline{\text { Age }}$} & 30s & 1 & 1 & 2 & NS \\
\hline & $40 \mathrm{~s}$ & 4 & 5 & 9 & \\
\hline & $50 \mathrm{~s}$ & 8 & 1 & 9 & \\
\hline & $60 \mathrm{~s}$ & 16 & 7 & 23 & \\
\hline & $70 \mathrm{~s}$ & 12 & 3 & 15 & \\
\hline & $80 \mathrm{~s}$ & 2 & 0 & 2 & \\
\hline & Mean & $63.5 \pm 10.5$ & $57.8 \pm 12.4$ & $61.9 \pm 11.2$ & \\
\hline \multirow[t]{2}{*}{ Age } & $<65$ years & 21 & 10 & 31 & NS \\
\hline & $\geq 65$ years & 22 & 7 & 29 & \\
\hline \multirow[t]{2}{*}{ Smoking } & No & 31 & 12 & 43 & NS \\
\hline & Yes & 12 & 5 & 17 & \\
\hline \multirow[t]{2}{*}{ Alcohol drinking } & No & 28 & 13 & 41 & NS \\
\hline & Yes & 15 & 4 & 19 & \\
\hline \multirow[t]{2}{*}{ H. pylori infection } & Negative & 34 & 16 & 50 & NS \\
\hline & Positive & 9 & 1 & 10 & \\
\hline \multirow[t]{4}{*}{ LA classification } & $\mathbf{A}$ & 18 & 11 & 29 & NS \\
\hline & B & 20 & 5 & 25 & \\
\hline & $\mathrm{C}$ & 4 & 1 & 5 & \\
\hline & D & 1 & 0 & 1 & \\
\hline \multirow[t]{5}{*}{ GERD duration } & $\leq 2$ months & 13 & 2 & 15 & $p=0.0004$ \\
\hline & $\leq 6$ months & 14 & 1 & 15 & \\
\hline & $\leq 12$ months & 1 & 5 & 6 & \\
\hline & $>12$ months & 4 & 6 & 10 & \\
\hline & Unknown & 11 & 3 & 14 & \\
\hline \multirow[t]{2}{*}{ Hernia } & No & 29 & 9 & 38 & NS \\
\hline & Yes & 14 & 8 & 22 & \\
\hline \multirow[t]{4}{*}{ Extent of hernia } & $\leq 1 \mathrm{~cm}$ & 15 & 5 & 20 & NS \\
\hline & $1-3 \mathrm{~cm}$ & 10 & 4 & 14 & \\
\hline & $>3 \mathrm{~cm}$ & 4 & 0 & 4 & \\
\hline & Unknown & 14 & 8 & 22 & \\
\hline \multirow[t]{2}{*}{ Complication } & No & 25 & 14 & 39 & NS \\
\hline & Yes & 18 & 3 & 21 & \\
\hline
\end{tabular}

result. 1) The acid-suppressing effects of rabeprazole $(20 \mathrm{mg}$ once daily) did not differ from those of omeprazole $(20 \mathrm{mg}$ once daily), as has been reported and may also have been affected by the CYP2C19 genotype, meaning that the drug was unable to produce an additional acid-suppressing effect. Contrary to expectations, our findings suggest that switching from omeprazole (20 mg once daily) to rabeprazole (20 mg once daily, the double dose), is not very effective as a therapeutic strategy for improving GERD symptoms. In this study, $92 \%$ of the patients refractory to treatment with rabeprazole at a dose of $20 \mathrm{mg}$ once daily exhibited an EM status, with an equal number of subjects being homo- and heterozygous EM; thus, the rabeprazole treatment may have been affected by the CYP2C19 genotype in terms of symptom improvement. 2) Taking into account the findings of past reports of the acid-suppressing effects of rabeprazole
(20 mg once daily), acid regurgitation may be involved to a minor extent in the condition of patients with symptoms lasting after the administration of omeprazole at a dose of $20 \mathrm{mg}$ once daily for $\geq 8$ weeks. That is to say, factors other than acid, such as psychometric factors, as discussed below, may play a significant role.

The results of the FSSG and HADS questionnaires showed that the patients with persistent GERD symptoms had specific background factors, including a longer disease duration and more severe anxiety and/or depression. Two studies in Japan used the FSSG alone to evaluate background factors related to PPI-refractory symptoms. In one study, 101 GERD patients (28 RE patients, 73 NERD patients) were treated with rabeprazole at a dose of $10 \mathrm{mg}$ once daily for four weeks, and, if their symptoms were not relieved, the rabeprazole dose was doubled every two 


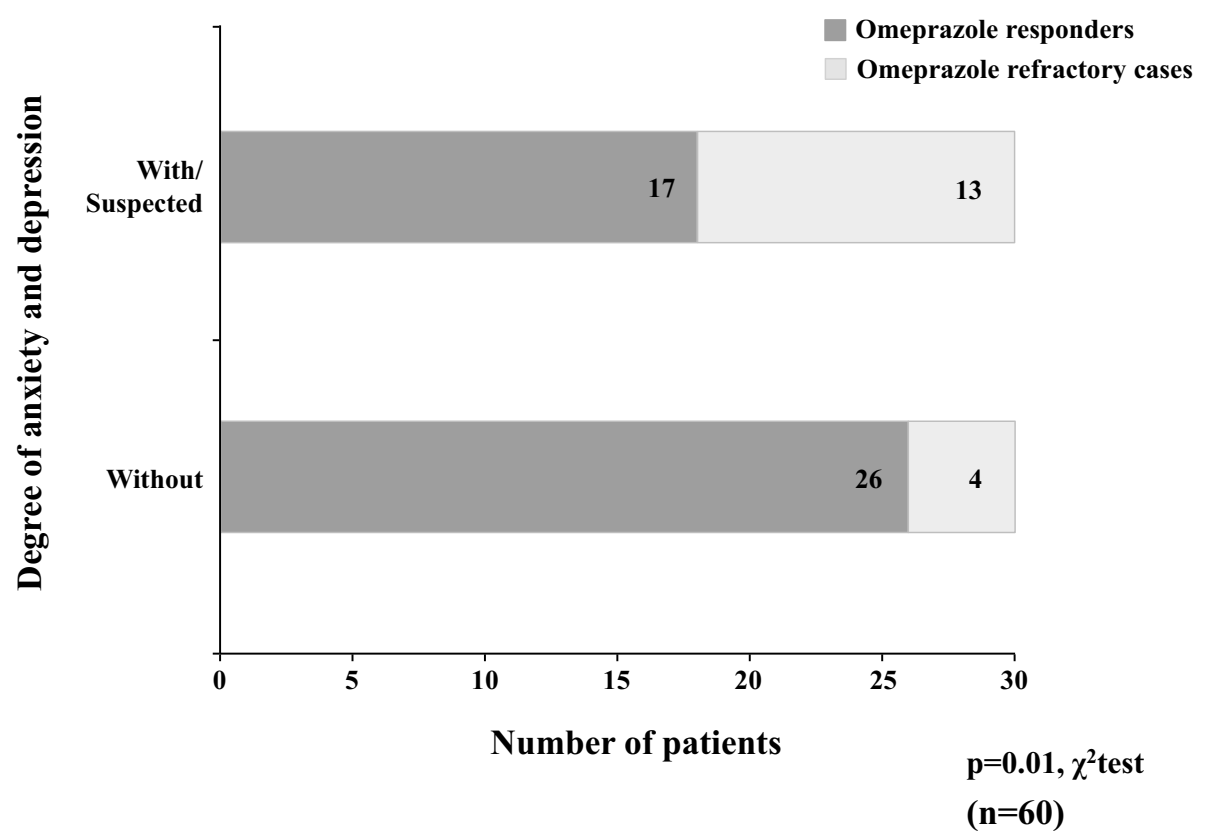

Figure 3. Therapeutic effect of treatment with omeprazole at a dose of $20 \mathrm{mg}$ once daily according to the presence/absence of psychometric factors. The more severe the anxiety and/or depression, the larger the number of patients found to be refractory to treatment with omeprazole at a dose of $20 \mathrm{mg}$ once daily.

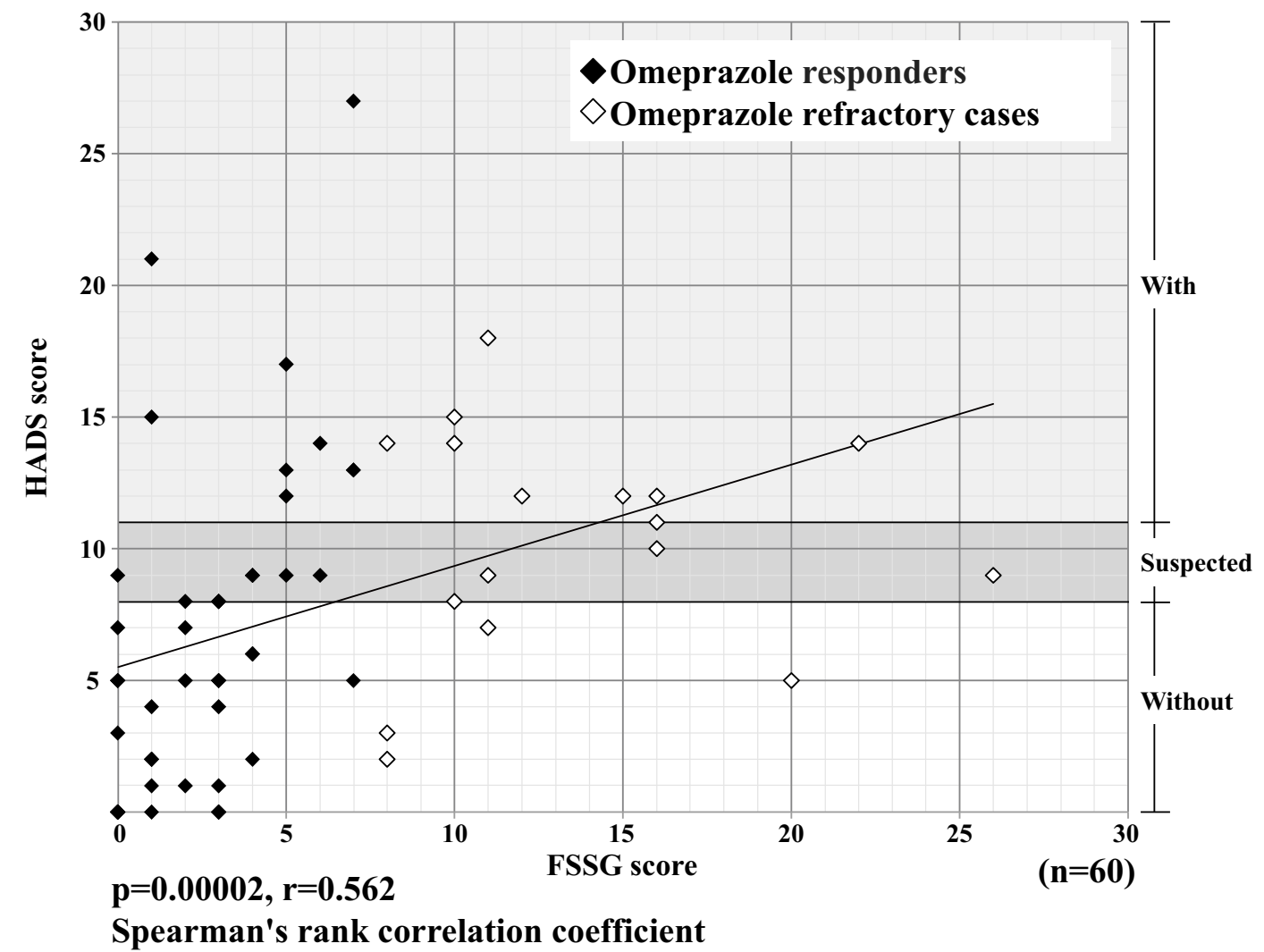

Figure 4. Relationship between the FSSG score and HADS score following treatment with omeprazole at a dose of $20 \mathrm{mg}$ once daily. A significant correlation was observed between the FSSG and HADS scores: the higher the FSSG score, the higher the HADS score.

weeks. Background factors related to heartburn relief or persistence were subsequently assessed over a total of eight weeks (22). Consequently, the rate of complete heartburn relief in the RE patients was $67.9 \%, 84.0 \%$ and $91.7 \%$ after 
Table 2. Background Factors of Patients Treatment with Rabeprazole at a Dose of $20 \mathrm{mg}$ Once Daily

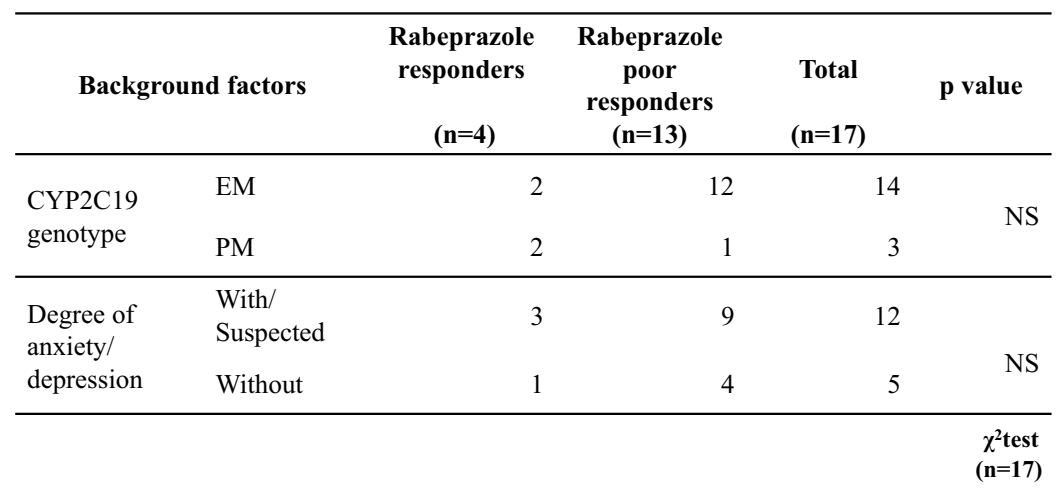
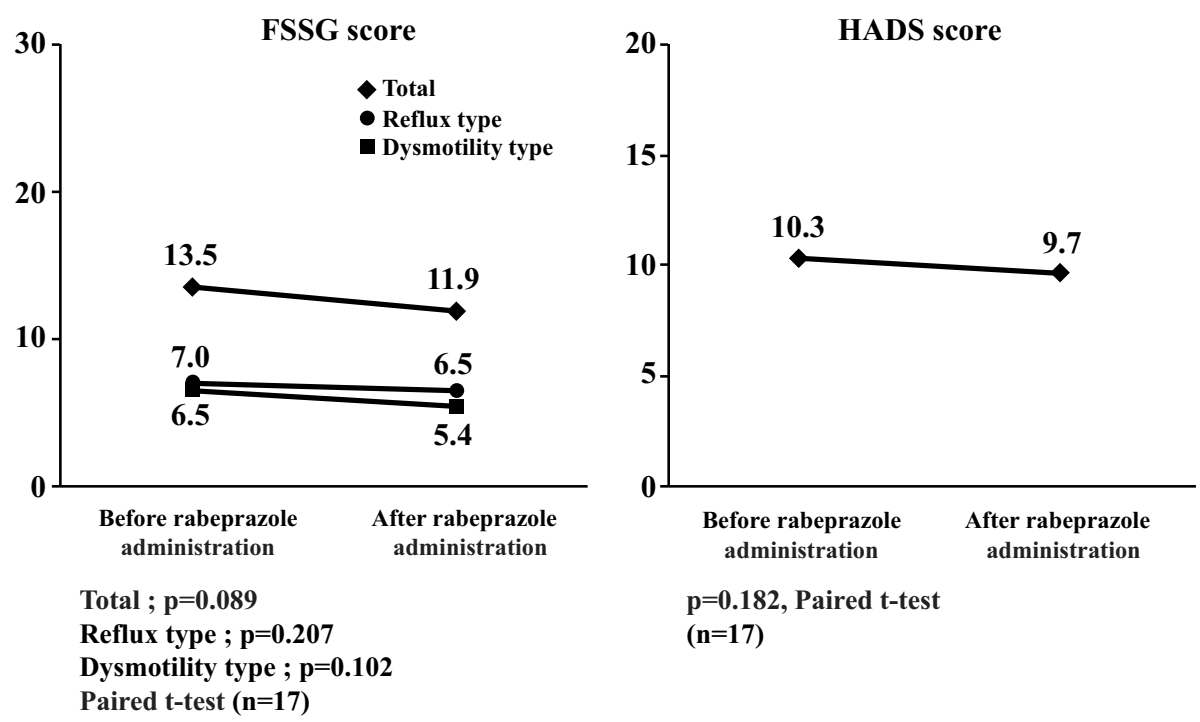

Figure 5. Changes in the FSSG and HADS scores from before to after treatment with rabeprazole at a dose of $20 \mathrm{mg}$ once daily. No significant differences were noted in the FSSG or HADS scores from before to after the administration of rabeprazole at a dose of $20 \mathrm{mg}$ once daily.

four, six and eight weeks, respectively, and more than $90 \%$ of the patients achieved complete symptom relief following the administration of rabeprazole $40 \mathrm{mg}$ once daily. In contrast, the rate of complete heartburn relief in the NERD patients was $42.5 \%, 61.5 \%$ and $68.9 \%$, respectively. Furthermore, background factors associated with resistance to rabeprazole (10 mg once daily, the standard dose in Japan) were a female sex, non-smoking, frequent heartburn, low FSSG Q4 score (a question about acid reflux: "subconsciously rubbing the chest"), high Q7 score ("unusual sensation in the throat") and high Q3 score (a question about dysmotility: "a heavy stomach after a meal"). Meanwhile, background factors associated with resistance to rabeprazole (20 $\mathrm{mg}$ once daily, a double dose in Japan) included frequent heartburn and a high Q7 score. In the other study, the PPI non-response rate and background factors were assessed among 467 GERD patients (118 RE patients, 349 NERD patients), including RE patients who received rabeprazole at a dose of $10 \mathrm{mg}$ once daily, lansoprazole at a dose of $30 \mathrm{mg}$ once daily or omeprazole at a dose of $20 \mathrm{mg}$ once daily and
NERD patients who received a half dose of lansoprazole or omeprazole for two weeks (23). Consequently, the PPI nonresponse rates in the RE and NERD patients were $42.4 \%$ and $52.7 \%$, respectively, and factors for PPI non-response in both patient groups were a younger age and higher score on the FSSG dysmotility question Q8 ("fullness while eating").

The HADS is a self-administered questionnaire developed to evaluate states of depression and anxiety independently from physical symptoms (24). We searched PubMed for studies that used the HADS alone in GERD patients and we found none conducted in Japan and two performed outside Japan. One study had evaluated GERD patients with sleep disturbances and emotional dysfunction (25), while the other had assessed GERD patients with asthma (26). Both studies described a relationship between symptoms and psychometric factors; however, the analyses were conducted in GERD patients with other diseases and thus cannot be directly compared with our findings. The present study is the first to report a significant correlation between the FSSG and HADS in RE patients with PPI-refractory GERD symptoms. 
In our study, the mean HADS total score before and after switching to rabeprazole (20 mg once daily) was 10.3 and 9.7, respectively, both of which are lower than that reported in the two studies discussed above. In addition, the level of anxiety and/or depression in this study was assessed as mild based on the HADS score, and no changes in the scores were observed as a result of switching the PPI treatment. Therefore, there is a possibility that psychometric factors were strongly involved in producing PPI-refractory symptoms in our study population. Our results suggest that the use of both FSSG and HADS to evaluate the level of anxiety and depression in RE patients makes it easier to predict whether a patient will be PPI-refractory due to psychometric factors. In addition, the fact that the PPI-refractory patients had higher HADS scores suggests the possibility that some of these patients also had a preexisting functional gastrointestinal disorder, such as functional dyspepsia, which is strongly associated with psychological stress.

These observations suggest the possibility that, even in patients diagnosed with RE, switching to treatment with a double dose of a PPI does not show promise in subjects with persistent PPI-refractory symptoms. Potential reasons for this result include 1) the presence of patients in whom psychometric factors play a major role, as evaluated according to the HADS, and 2) the presence of cases that require stronger acid suppression than that achieved with a double dose of PPI therapy. In addition, we cannot rule out alkaline reflux or hyperesthesia as a cause of persistent symptoms. However, in this study, endoscopic examinations and alkaline reflux tests were not performed following the administration of omeprazole or the switch to rabeprazole. Therefore, we were not able to determine the causes of the remaining symptoms in individual PPI-refractory patients. The limitations of our study include the fact that the sample of patients considered to be PPI-refractory and who switched drugs was small and that CYP2C19 genotyping was performed only in this small subset.

\section{Conclusion}

The results of this study indicate that switching from omeprazole (20 mg once daily) to rabeprazole (20 mg once daily) is not an effective therapeutic strategy for improving PPI-refractory GERD symptoms, taking into consideration possible psychometric factors and the presence of patients who require stronger acid suppression than that achieved with a double dose of PPIs for PPI-refractory GERD symptoms.

\section{The authors state that they have no Conflict of Interest (COI).}

\section{Acknowledgement}

We wish to thank Interscience Co., Ltd. for providing editorial support.

\section{Financial Support}

Financial support for the preparation of this paper was provided by AstraZeneca K.K. Osaka, Japan according to the publication support agreement.

\section{References}

1. Vakil N, van Zanten SV, Kahrilas P, Dent J, Jones R. Global Consensus Group. The Montreal definition and classification of gastroesophageal reflux disease: a global evidence-based consensus. Am J Gastroenterol 101: 1900-1920, 2006.

2. Fock KM, Talley NJ, Fass R, et al. Asia-Pacific consensus on the management of gastroesophageal reflux disease: update. J Gastroenterol Hepatol 23: 8-22, 2008.

3. Katz PO, Gerson LB, Vela MF. Guidelines for the diagnosis and management of gastroesophageal reflux disease. Am J Gastroenterol 108: 308-328, 2013.

4. Fujiwara Y, Arakawa T. Epidemiology and clinical characteristics of GERD in the Japanese population. J Gastroenterol 44: 518-534, 2009.

5. Gastroesophageal reflux disease (GERD) treatment guideline, compiled by the Japanese Society of Gastroenterology. Nankodo, Tokyo, 2010: 44-61.

6. Adachi K, Komazawa Y, Fujishiro H, et al. Nocturnal gastric acid breakthrough during the administration of rabeprazole and ranitidine in Helicobacter pylori-negative subjects: effects of different regimens. J Gastroenterol 38: 830-835, 2003.

7. Sugimoto M, Furuta T, Shirai N, et al. Different dosage regimens of rabeprazole for nocturnal gastric acid inhibition in relation to cytochrome P450 2C19 genotype status. Clin Pharmacol Ther 76: 290-301, 2004.

8. Shimatani $T$, Inoue $M$, Kuroiwa $T$, et al. Acid-suppressive effects of rabeprazole, omeprazole, and lansoprazole at reduced and standard doses: a crossover comparative study in homozygous extensive metabolizers of cytochrome P450 2C19. Clin Pharmacol Ther 79: 144-152, 2006.

9. Adachi K, Komazawa Y, Mihara T, et al. Administration of $\mathrm{H}_{2}$ receptor antagonist with proton pump inhibitor is effective for longterm control of refractory reflux esophagitis. J Clin Gastroenterol 38: 297-298, 2004.

10. Madan K, Ahuja V, Kashyap PC, Sharma MP. Comparison of efficacy of pantoprazole alone versus pantoprazole plus mosapride in therapy of gastroesophageal reflux disease: a randomized trial. Dis Esophagus 17: 274-278, 2004.

11. Martin-Merino E, Ruigómez A, Garcia Rodríguez LA, Wallander MA, Johansson S. Depression and treatment with antidepressants are associated with the development of gastro-oesophageal reflux disease. Aliment Pharmacol Ther 31: 1132-1140, 2010.

12. Wiklund I, Carlsson R, Carlsson J, Glise H. Psychological factors as a predictor of treatment response in patients with heartburn: a pooled analysis of clinical trials. Scand J Gastroenterol 41: 288293, 2006.

13. Kusano M, Shimoyama Y, Sugimoto S, et al. Development and evaluation of FSSG: frequency scale for the symptoms of GERD. J Gastroenterol 39: 888-891, 2004.

14. Zigmond AS, Snaith RP. The hospital anxiety and depression scale. Acta Psychiatr Scand 67: 361-370, 1983.

15. Beth D, Robert GT. Basic \& Clinical Biostatistics Fourth Edition. Apleton \& Lange, USA, 2004.

16. Dekkers CP, Beker JA, Thjodleifsson B, Gabryelewicz A, Bell NE, Humphries TJ. Double-blind comparison [correction of Double-blind, placebo-controlled comparison] of rabeprazole 20 $\mathrm{mg}$ vs. omeprazole $20 \mathrm{mg}$ in the treatment of erosive or ulcerative gastro-oesophageal reflux disease. The European Rabeprazole Study Group. Aliment Pharmacol Ther 13: 49-57, 1999.

17. Chiba N, De Gara CJ, Wilkinson JM, Hunt RH. Speed of healing 
and symptom relief in grade II to IV gastroesophageal reflux disease: a meta-analysis. Gastroenterology 112: 1798-1810, 1997.

18. Higuchi K, Joh T, Nakada K, Haruma K. Is proton pump inhibitor therapy for reflux esophagitis sufficient? : a large real-world survey of Japanese patients. Intern Med 52: 1447-1454, 2013.

19. McColl E, Junghard O, Wiklund I, Revicki DA. Assessing symptoms in gastroesophageal reflux disease: how well do clinicians' assessments agree with those of their patients?. Am J Gastroenterol 100: 11-18, 2005.

20. Dean BB, Gano AD Jr, Knight K, Ofman JJ, Fass R. Effectiveness of proton pump inhibitors in nonerosive reflux disease. Clin Gastroenterol Hepatol 2: 656-664, 2004.

21. Saitoh T, Otsuka H, Kawasaki T, et al. Influences of CYP2C19 polymorphism on recurrence of reflux esophagitis during proton pump inhibitor maintenance therapy. Hepatogastroenterology 56: 703-706, 2009.

22. Furuta $T$, Shimatani $T$, Sugimoto $M$, et al. Investigation of pretreatment prediction of proton pump inhibitor (PPI)-resistant pa- tients with gastroesophageal reflux disease and the dose escalation challenge of PPIs-TORNADO study: a multicenter prospective study by the Acid-Related Symptom Research Group in Japan. J Gastroenterol 46: 1273-1283, 2011.

23. Miyamoto M, Manabe N, Haruma K. Efficacy of the addition of prokinetics for proton pump inhibitor (PPI) resistant non-erosive reflux disease (NERD) patients: significance of frequency scale for the symptom of GERD (FSSG) on decision of treatment strategy. Intern Med 49: 1469-1476, 2010.

24. Zigmond AS, Snaith RP. The hospital anxiety and depression scale. Psychiatr Scand 67: 361-370, 1983.

25. Kim JY, Kim N, Seo PJ, et al. Association of sleep dysfunction and emotional status with gastroesophageal reflux disease in Korea. J Neurogastroenterol Motil 19: 344-354, 2013.

26. Cheung TK, Lam B, Lam KF, et al. Gastroesophageal reflux disease is associated with poor asthma control, quality of life, and psychological status in Chinese asthma patients. Chest 135: 11811185, 2009.

(C) 2015 The Japanese Society of Internal Medicine http://www.naika.or.jp/imonline/index.html 